EUROPEAN JOURNAL OF PURE AND APPLIED MATHEMATICS

Vol. 14, No. 4, 2021, 1108-1111

ISSN 1307-5543 - ejpam.com

Published by New York Business Global

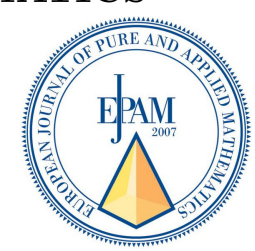

\title{
On Eigenvectors of Nilpotent Lie Algebras of Linear Operators
}

\author{
Morris W. Hirsch ${ }^{1,2, *}$, Joel W. Robbin ${ }^{2}$ \\ ${ }^{1}$ Department of Mathematics, University of California at Berkeley, Berkeley, CA 94720- \\ 384, USA \\ ${ }^{2}$ Department of Mathematics, University of Wisconsin at Madison, WI 53706, USA
}

\begin{abstract}
We give a condition ensuring that the operators in a nilpotent Lie algebra of linear operators on a finite dimensional vector space have a common eigenvector.
\end{abstract}

2020 Mathematics Subject Classifications: 22E25, 22E60, 47C05

Key Words and Phrases: Nilpotent, Lie algebras of Lie groups, Linear operators in algebras

\section{Introduction}

Throughout this paper $V$ is a vector space of positive dimension over a field $f$ and $\gg$ is a nilpotent Lie algebra over $f$ of linear operators on $V$. An element $u \in V$ is an eigenvector for $S \subset \gg$ if $u$ is an eigenvector for every operator in $S$. If $V$ has a basis $\left(e_{1}, \ldots, e_{n}\right)$ representing each element of $\gg$ by an upper triangular matrix, then $e_{1}$ is an eigenvector for $\gg$. Such a basis exists when $f$ is algebraically closed and $\gg$ is solvable (Lie's Theorem), and also when every element of $\gg$ is a nilpotent operator (Engel's Theorem). Our results are further conditions guaranteeing existence of eigenvectors.

The minimal and characteristic polynomials of a linear operator $A$ on $V$ are denoted respectively by $\pi_{A}, \mu_{A} \in f[t]=$ the ring of polynomials over $f$. The cardinality of a set $S$ is written $\# S$.

Let $k$ be a Galois extension field of $f$ of degree $d:=[k: f]$, and define $\mathbf{M} \subset$ to be the additive monoid generated by zero and the prime divisors $d$.

Consider the conditions:

(C1) $\mu_{A}$ splits in $k$ for every $A \in \gg$

(C2) $\operatorname{dim} V \notin \mathbf{M}$

*Corresponding author.

DOI: https://doi.org/10.29020/nybg.ejpam.v14i4.4086

Email addresses: mwhirsch@chorus.net (M. W. Hirsch), robbin@math.wisc.edu (J. W. Robbin) 


\section{Results}

Our main result is:

Theorem 1. If(C1) and(C2) hold then $\gg$ has an eigenvector.

The proof is preceded by some applications.

When(C1) holds, Theorem 1 shows that there is an eigenvector in every invariant subspace whose dimension is not in $\mathbf{M}$. This is exploited to yield the following two results:

Corollary 1. If a nilpotent Lie algebra of linear operators on $n$ does not have an eigenvector, every nontrivial invariant subspace has odd dimension.

Proof. When $f$ is the real field and $k$ is the complex field, $\mathbf{M}$ consists of the positive even integers.

Corollary 2. Let(C1) hold. Assume $\gg$ preserves a direct sum decomposition $V=\oplus_{i} W_{i}$, and let $D \subset$ denote the set of dimensions of the subspaces $W_{i}$.

(i) If $\gg$ does not have an eigenvector then $D \subset \mathbf{M}$.

(ii) If $V^{\prime} \subset V$ is a maximal subspace spanned by eigenvectors of $\gg$ then $\operatorname{dim}\left(V^{\prime}\right) \geq$ $\#\{D \backslash \mathbf{M}\}$.

Proof. Assertion (i) follows from Theorem 1. To prove (ii) order the $W_{i}$ so that $W_{1}, \ldots, W_{m}$ are the only summands whose dimensions are not in $\mathbf{M}$. For each $j \in$ $\{1, \ldots, m\}$ we choose an eigenvector $e_{j} \in W_{j}$ by Theorem 1 . The $e_{j}$ are linearly independent and belong to $V^{\prime}$ by maximality of $V^{\prime}$, whence (ii).

Example 1. Assume $n \notin \mathbf{M}$ and let $\alpha \in f[t]$ be a monic polynomial that splits in $k[t]$. Denote by $A(\alpha)$ the set of $n \times n$ matrices $T$ over $f$ such that $\alpha(T)=0$. Then every pairwise commuting family $T \subset A(\alpha)$ has an eigenvector in $f^{n}$. This follows from Theorem 1 applied to the Lie algebra $\gg$ of linear operators on $f^{n}$ generated by $T$. Being abelian, $\gg$ can be triangularized over $k$, hence $(\boldsymbol{C 1})$ holds.

Example 2. The assumption that $n \in \mathbf{M}$ is essential to Theorem 1. For instance, take $f=, k=, V=2$. The abelian Lie algebra of $2 \times 2$ of real skew symmetric matrices. does not have an eigenvector in 2 .

Example 3. The hypothesis of Theorem 1 cannot be weakened to $\gg$ being merely solvable. For a counterexample with $f=, k=$, take $\gg$ to be the solvable 3-dimensional real Lie algebra with basis $(X, U, V)$ such that $[X, U]=-V,[X, V]=U,[U, V]=0$.

A Lie algebra $\beta$ over $f$ is supersolvable if the spectrum of the linear map ad $A: \beta \rightarrow \beta$ lies in $f$ for all $A \in \beta$. If $\beta$ is not supersolvable it need not have an eigenvector, as is shown by Example 3. We don't know if Theorem 1 extends to supersolvable Lie algebras, except for the following special case: 
Theorem 2. A supersolvable Lie algebra $\beta$ of linear transformations of 3 has an eigenvector.

Proof. Lacking an algebraic proof, we use a dynamical argument. Let $G \subset G L(3$, be the connected Lie subgroup having Lie algebra $\beta$. The natural action of $G$ on the projective plane $\boldsymbol{\Upsilon} 2$ of lines in 3 through the origin fixes some $L \in \mathbb{q} 2$. This follows from supersolvability because $\operatorname{dim}(\boldsymbol{\Upsilon} 2)=2$, the action on $\Upsilon 2$ is effective and analytic, and the Euler characteristic of $\ 2$ is nonzero (Hirsch \& Weinstein [1]). The nonzero points of $L$ are eigenvectors for $\beta$.

\subsection{Proof of Theorem 1}

We rely on Jacobson's Primary Decomposition Theorem [2, II.4, Theorem 5]. This states that $V$ has a $\gg$-invariant direct sum decomposition $\oplus V_{i}$ where each primary component $V_{i}$ has the following property: For each $A \in \gg$ the minimal polynomial of $A \mid V_{i}$ is a prime power in $f[t]$.

Condition(C2) implies the dimension of some primary component is $\notin \mathbf{M}$. To prove Theorem 1 it therefore suffices to apply the following result to such a primary component:

Theorem 3. Assume(C1) and(C2). If $\pi_{A}$ is a prime power in $f[t]$ for each $A \in \gg$ then the following hold:

(a) $\pi_{A}(t)=\left(t-r_{A}\right)^{n}, r_{A} \in f$

(b) there is a basis putting $\gg$ in triangular form

Assertion (a) is equivalent to $\pi_{A}$ having a root $r_{A} \in f$. Therefore (a) follows from:

Lemma 1. Let $\alpha \in f[t]$ be a polynomial of degree $n$ that splits in $k[t]$. If $n \notin \mathbf{M}$ then $\alpha$ has a root in $f$, and the sum of the multiplicities of such roots is $\notin \mathbf{M}$.

Proof. Let $R \subset k$ denote the set of roots of $\pi$, and $R_{j} \subset R$ the set of roots of multiplicity $j$.

The Galois group $\Gamma$ has order $[k: f]$ and acts on $R$ by permutations. The cardinality of each orbit divides $[k: f]$, and $R \cap f$ is the set of fixed points of this action.

Each $R_{j}$ is a union of orbits, as is $R_{j} \backslash f$. It follows that $\#\left(R_{j} \backslash f\right) \in \mathbf{M}$.

Let $k \leq n$ denote the sum of the multiplicities of the roots that are not in $f$. Then

$$
k=\sum_{j=2}^{n} j \cdot \#\left(R_{j} \backslash f\right)
$$

Therefore $k \in \mathbf{M}$ because $\mathbf{M}$ is closed under addition.

By hypothesis $n \notin \mathbf{M}$, hence $n-k \notin \mathbf{M}$ and $n-k>0$. As $n-k$ is the sum of the multiplicities of the roots in $f$, the conclusion follows.

Now that (a) of Theorem 3 is proved, assertion (b) is a consequence of the following result: 
Lemma 2. Let be a nilpotent Lie algebra of linear operators on $V$. Assume that for all $A \in$ there exists $r_{A} \in f$ such that $\pi_{A}(t)=\left(t-r_{A}\right)^{n}$.

Then $V$ has a basis putting in triangular form.

Proof. Every $A \in$ can be written uniquely as $r_{A} I+N_{A}$ with $N_{A}$ nilpotent and $I$ the identity map of $V$. It is easy to see that the set comprising the $N_{A}$ is closed under commutator brackets. Therefore $V$ has a basis triangularizing all the $N_{A}$ (Jacobson $[2$, II.2, Theorem $\left.1^{\prime}\right]$ ), and such a basis triangularizes .

This completes the proof of Theorem 1.

\section{References}

[1] M Hirsch and A Weinstein. Fixed points of analytic actions of supersoluble Lie groups on compact surfaces. Ergod. Th. Dyn. Sys., 21(6):1783-1787, 2001.

[2] N Jacobson. Lie Algebras. Interscience Tracts in Pure Mathematics No. 10, John Wiley, New York, 1962. 